\title{
Use of the ClearSight ${ }^{\circ}$ System for Continuous Noninvasive Hemodynamic Monitoring during Heart Valve Interventions: Review of the Literature and Single-Site Experience
}

\author{
George Gellert, $\mathbf{M D},{ }^{1}$ Peter Bramlage, $\mathrm{MD}^{2}$
}

${ }^{1}$ Interventional Echocardiography, Structural Heart Program, Banner - University Medical Center Phoenix, Phoenix, AZ, USA; 2Institute for Pharmacology and Preventive Medicine, Cloppenburg, Germany

\section{ABSTRACT}

During interventional and structural cardiology procedures, such as mitral valve (MitraClip, BMV), aortic valve (TAVR, BAV), tricuspid valve (MitraClip), left atrial appendage (Watchman, Lariat), atrial septum (ASD/PFO closure), and coronary artery intervention (high-risk PCI), among others, patients are at a high risk of hemodynamic instability and require continuous monitoring. This is conventionally achieved through arterial catheterization and transpulmonary thermodilution. However, such invasive techniques are time-consuming and have been associated with steep learning curves, vascular complications, and increased risk of infection. In line with the ongoing simplification and improvement of the catheter-based valve intervention, it is logical to investigate the effectiveness of continuous noninvasive hemodynamic monitoring in this setting. Over the last 2 years, our team has performed over 400 valve procedures with continuous hemodynamic monitoring via the noninvasive ClearSight system. This system is based on a finger-cuff and automated volume-clamp technology integrated into a simplified clinical platform (EV1000 NI). Although current evidence suggests that the technology results in slight differences in arterial pressure (AP) and cardiac output $(\mathrm{CO})$ relative to the current, commercially available, invasive approaches, we have found the bias to be acceptable. Both the noninvasive and the invasive approaches have the same percentage of error when compared to the true $\mathrm{CO}$ and provide beat-by-beat detection of acute changes facilitating shorter response times. In addition to $\mathrm{AP}$ and $\mathrm{CO}$, the system provides up-to-date information on stroke volume (SV), stroke volume variation (SVV), and systemic vascular resistance, which can be useful in aiding decision-making and provide better postoperative outcomes, such as shorter length of stay (LOS), decreased postoperative infection, decreased postoperative arrhythmia, decreased postoperative renal failure, decreased postoperative congestive heart failure (CHF), and decreased readmission.

Received August 22, 2018; received in revised form September 29, 2018; accepted October 1, 2018.

Correspondence: George Gellert, MD, Interventional Echocardiography, Structural Heart Program, Banner - University Medical Center Phoenix, 111 E McDowell Rd, Phoenix, AZ 85006, USA; 1-602-689-6535 (e-mail: georgegellert@gmail.com).
Additionally, the simplicity of the system setup has translated into a time saving of up to 3 hours per day, allowing one team to perform an additional 2 to 3 valve interventions without moving rooms. Moving forward, a formal study comparing patient outcomes and cost-effectiveness between invasive and noninvasive hemodynamic monitoring techniques in valve replacement would be insightful.

\section{INTRODUCTION}

During interventional and structural cardiology procedures, such as mitral valve (MitraClip [Abbott, Abbott Park, IL, USA], BMV), aortic valve (TAVR, BAV), tricuspid valve (MitraClip), left atrial appendage (Watchman [Boston Scientific, Marlborough, MA, USA], Lariat [SentreHEART, Redwood City, CA, USA]), atrial septum (ASD/PFO closure), and coronary artery interventions (high-risk PCI), among others, patients are at a high risk of hemodynamic instability and require continuous monitoring. During aortic valve intervention, for example, this is due to the rapid pacing used to reduce cardiac output for balloon dilation and valve implantation [Fassl 2009]; the potential for complications such as aortic regurgitation, left ventricular or aortic rupture, cardiac tamponade, and new-onset arrhythmias [Giustino 2014; Dolmatova 2017]; and the high prevalence of carotid, aortic, valvular, coronary, and peripheral vascular diseases commonly found in patients with aortic stenosis [Cattaneo 2010]. Because insufficient or delayed hemodynamic management during the procedure has been associated with a higher likelihood of mortality [Tamburino 2011], close monitoring of cardiac parameters throughout the intervention is paramount. Conventionally, hemodynamic monitoring is accomplished through the placement of invasive arterial/central venous catheters. However, catheterization is time-consuming, requires a high level of technical skill, and can result in complications such as vascular injury, thrombosis, and infection [Scheer 2002]. Furthermore, use of mean AP (MAP) alone to detect acute hemodynamic alterations is unreliable, because such changes are generally related to blood flow rather than alterations in vasotone and physiological adaptation may maintain MAP despite variations in cardiac output (CO) [Petzoldt 2015]. The invasive alternative, transpulmonary thermodilution, requires calibration with cold fluid boluses, creating an additional fluid load. Given that valve procedures are becoming ever more simplified, with movement towards 


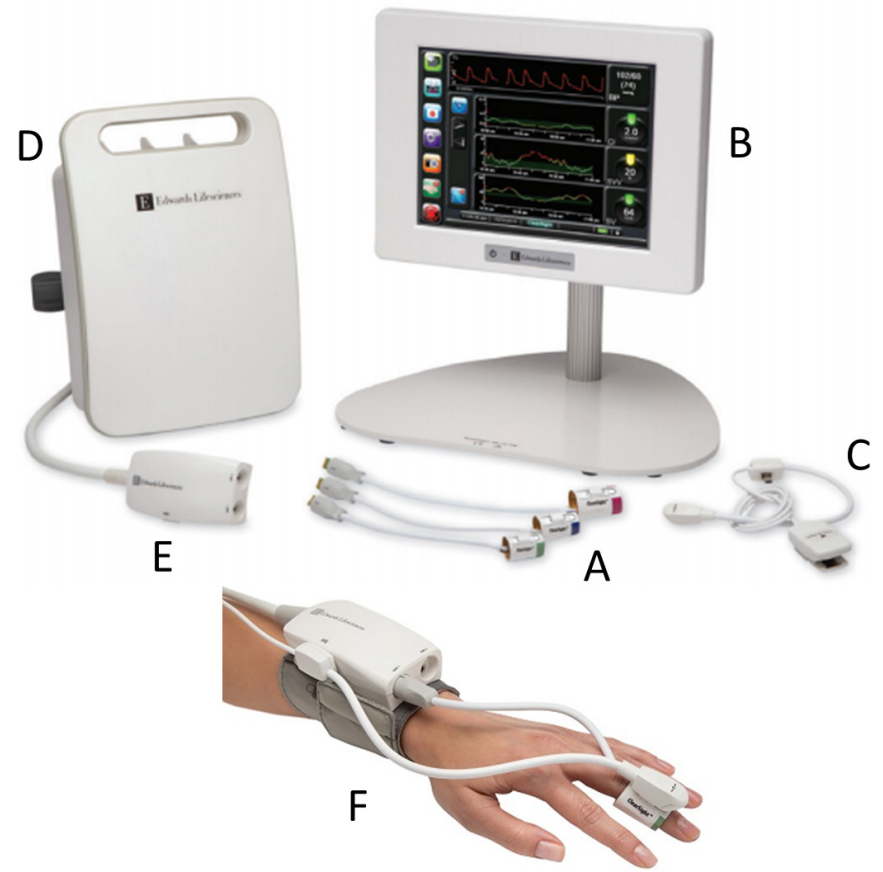

Figure 1. ClearSight system components (Edwards Lifesciences, with permission). (A) Finger cuff (3 sizes available); (B) EV1000 NI clinical platform for clear data presentation; (C) the hydrostatic pressure changes due to difference in height between the finger and heart are compensated by the HRS; (D) pump unit; (E) pressure controller; (F) correct placement of a single finger cuff, heart reference sensor, and pressure controller on a patient.

greater use of conscious sedation [Landes 2017; Lee 2017], a logical next step is to reduce the reliance on such invasive monitoring techniques with the aim of improving patient experience and increasing center productivity.

A number of noninvasive approaches to continuous hemodynamic monitoring have been developed to address the limitations of invasive monitoring. These include thoracic bioimpedance/bioreactance, pulse wave transit time based on electrocardiogram/plethysmography, and pulse contour analysis based on finger-cuff pressure devices [Joosten 2017]. The ClearSight system from Edwards Lifesciences (Irvine, CA, US) is based on the latter principle and allows real-time beat-to-beat monitoring of multiple cardiac parameters [Kalmar 2013]. The Nexfin® technology underlying the ClearSight system has been evaluated in a number of clinical settings [Stover 2009; Bogert 2010; Martina 2010; Van de Vijver 2011; Broch 2012; Fischer 2012; Kalmar 2012; Martina 2012; Monnet 2012; Ameloot 2013; Broch 2013; BubenekTurconi 2013; Hohn 2013; Ameloot 2014; Hofhuizen 2014; Maass 2014; Vos 2014; Weiss 2014; Balzer 2016; de Wilde 2016; Heusdens 2016; Schraverus 2016; Berkelmans 2018; Sperna Weiland 2018]; however, its specific impact on valve procedures remains to be determined. Herein, we present insights from the Banner - University Medical Center Phoenix (Phoenix, AZ, USA), an experienced site performing valve interventions, and contextualize our observations within the

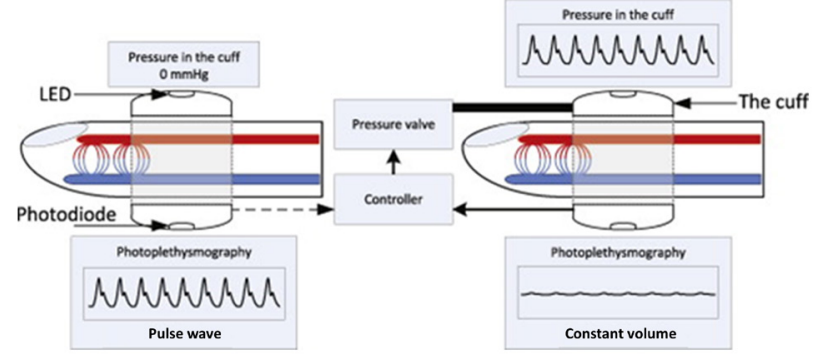

Figure 2. Schematic representation of the Nexfin volume-clamp principle. Figure source: [Peter 2014]. The finger cuff inflates and deflates over the course of a cardiac cycle to counter the pressure exerted by the pulse wave in the finger artery, such that the volume in the finger artery remains constant. The pressure in the cuff may then be considered equal to arterial pressure. This is periodically recalibrated on the basis of the shape of the plethysmogram by the Physiocal ${ }^{T M}$ algorithm. The resulting pulse wave is then reproduced at a brachial level. Finally, an afterload model is used to determine other parameters such as stroke volume (SV), stroke volume variation (SVV), and cardiac output (CO) [Kalmar 2013].

existing literature.

\section{THE CLEARSIGHT SYSTEM}

The ClearSight system (Figure 1) employs Nexfin technology, which was originally developed and introduced onto the market by BMEYE (the Netherlands) in 2007. As such, it also relies on the Physiocal ${ }^{\mathrm{TM}}$ autocalibration unloading algorithm in combination with the volume-clamp technique, but has been integrated into a simplified clinical platform (EV1000 NI) for clearer, more versatile visual support. An in-depth description of Nexfin technology can be found in Kalmar et al, 2013 [Kalmar 2013], whereas a schematic representation is provided in Figure 2.

Briefly, ClearSight consists of a disposable pneumatic finger cuff that inflates and deflates over the course of a cardiac cycle according to signals from an integrated photoplethysmography sensor. This sensor detects fluctuations in infrared light absorption by the red blood cells (RBCs) that reflect volume changes in the finger artery during pulsation; absorption (and therefore finger blood volume) increases during systole and falls during diastole. The resulting absorption curve is therefore an indication of arterial blood volume. The aim of dynamic cuff inflation/deflation is to apply the exact pressure required to counteract the pressure changes in the finger artery throughout the cardiac cycle, preventing changes in the diameter of the vessel and maintaining a constant finger volume (volume-clamp technique) [Raggi 2017]. A flat absorption curve indicates that this state has been reached, at which point the counterpressure exerted by the cuff over the cardiac cycle may be considered equivalent to that exerted by the pulse wave in the finger artery. An algorithm reconstructs this pressure curve at the brachial level [Kalmar 2013]; no arm cuff calibration is required. Subsequently, pulse contour analysis and a 3-element Windkessel model for cardiac afterload can be used to quantify related 
Evidence for the Accuracy and Precision of Nexfin Technology Compared to Invasive Monitoring Techniques* $\dagger$

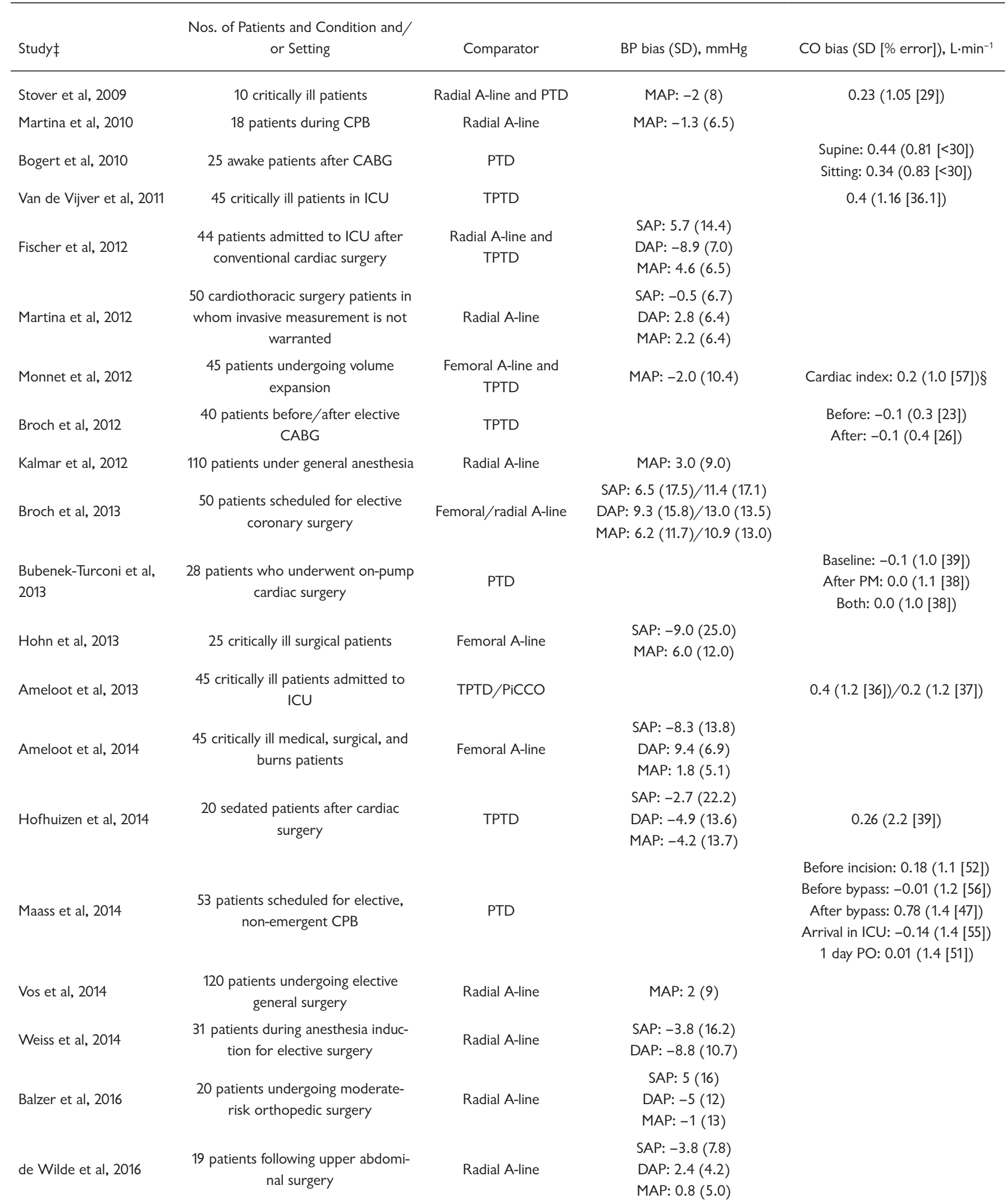


Evidence for the Accuracy and Precision of Nexfin Technology Compared to Invasive Monitoring Techniques* $†$ [Continued]

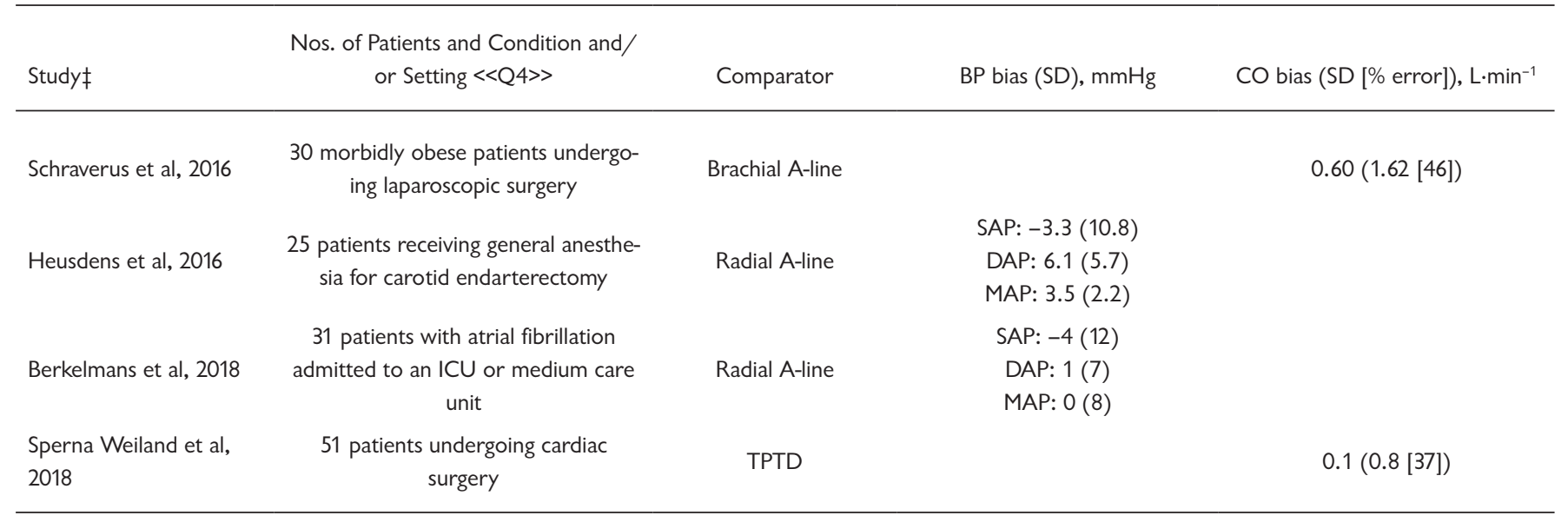

*Biases are based on Bland-Altman analysis [Bland 1986]. For some studies, only limits of agreement were presented, in which case SD was calculated by dividing the mean of the 2 limits by 1.96 .

$\nmid B$, blood pressure; SD, standard deviation; CO, cardiac output; A-line, arterial line; PTD, pulmonary thermodilution; MAP, mean arterial pressure; CPB, cardiopulmonary bypass; CABG, coronary artery bypass graft; ICU, intensive care unit; TPTD, transpulmonary thermodilution; SAP, systolic arterial pressure; DAP, diastolic arterial pressure; PM, preload-modifying maneuver; PiCCO, pulse contour cardiac output; PO, postoperatively.

$\ddagger$ Each entry in the "Study" column refers to the corresponding reference in the "References." Also, note that each date given in this table is the date of the reference and is not necessarily the date of the study.

$\S U n i t: L \cdot \min ^{-1} \cdot(\mathrm{m} 2)^{-1}$.

variables such as stroke volume (SV), stroke volume index (SVI), stroke volume variation (SVV), CO, and systemic vascular resistance (SVR) on a beat-by-beat basis. To compensate for ongoing changes in vascular physiology that may also contribute to arterial volume, the finger cuff is automatically recalibrated every 5-70 beats in response to information from the photoplethysmography sensor [Stenglova 2017]. This means that BP changes due to variations in cardiac parameters are immediately and easily discernible from confounding influencers.

\section{ACCURACY AND PRECISION COMPARED TO INVASIVE HEMODYNAMIC MONITORING SYSTEMS}

The accuracy and precision of hemodynamic data from Nexfin-based devices have been evaluated relative to invasive A-line and thermodilution monitoring strategies in multiple small-scale studies across various clinical settings (Table) [Stover 2009; Bogert 2010; Martina 2010; Van de Vijver 2011; Broch 2012; Fischer 2012; Kalmar 2012; Martina 2012; Monnet 2012; Ameloot 2013; Broch 2013; Bubenek-Turconi 2013; Hohn 2013; Ameloot 2014; Hofhuizen 2014; Maass 2014; Vos 2014; Weiss 2014; Balzer 2016; de Wilde 2016; Heusdens 2016; Schraverus 2016; Berkelmans 2018; Sperna Weiland 2018]. In general, studies appear to report acceptable mean arterial pressure (MAP) biases when compared to the gold standard arterial catheter method [Stover 2009; Martina 2010; Fischer 2012; Martina 2012; Ameloot 2014; Hofhuizen 2014; de Wilde 2016; Heusdens 2016; Berkelmans 2018]. Therefore, the accuracy and precision values recorded for the Nexfin technology may be considered adequate for the purposes of perioperative monitoring in valve interventions, especially when taken in the context of its noninvasive advantage and the additional cardiac variables it provides. Indeed, almost all comparative studies report Nexfin arterial pressure biases that fall within $<10 \mathrm{mmHg}$ of the reference method [O'Brien 2010].

Whereas certain noninvasive devices have only been validated in sedated patients in normal sinus rhythm and arrhythmia may result in inaccuracies with many peripheral monitoring systems, it is of particular note that the Nexfin/ClearSight system is much less affected by this potential source of error. Maggi and colleagues evaluated the technology in 22 patients undergoing interventional electrophysiology procedures which are characterized by critical situations of hypotension triggered by tachyarrhythmia or by intermittent incremental ventricular temporary pacing [Maggi 2010]. They found that the output of the Nexfin was not affected. A linear correlation for a range of $\mathrm{BP}$ values from 41 to $190 \mathrm{mmHg}$ was found between noninvasive and intra-arterial BP among a total of 1055 beats from 3 patients who underwent simultaneous recordings with both methods (coefficient of correlation of $0.81, P<.0001)$.

Although conventional monitoring generally makes use of invasive pulmonary artery or transpulmonary thermodilution for determination of $\mathrm{CO}$, the ClearSight system allows both $\mathrm{BP}$ and $\mathrm{CO}$ readings to be performed simultaneously and noninvasively. Nexfin technology has been shown to meet the currently accepted percentage-of-error requirements on a number of occasions both in awake patients [Stover 2009; 
Bogert 2010] and during coronary artery surgery [Broch 2012] (data presented in Table).

Two years ago, we carried out our own assessment of the accuracy and reliability of the ClearSight system during valve interventions. Over the course of several months, approximately 100 patients undergoing valve replacement at our site were subjected to simultaneous invasive (femoral A-line by implanter team, transpulmonary thermodilution, FloTrac [Edwards Lifesciences], and TEE) and noninvasive (ClearSight system) periprocedural hemodynamic monitoring. This revealed a striking similarity between the data produced by the 2 approaches, such that we have now switched to using the ClearSight system only during such procedures. Over the last 2 years, we have performed over 400 procedures with the ClearSight system and have found the reliability of data and patient outcomes to be extremely satisfactory. In particular, the ready availability of SV, SVI, SVV, CO, and SVR data (none of which are obtainable from just an A-line) provides additional useful information on which to base decision-making, with beat-by-beat monitoring allowing early detection of hemodynamic changes and shorter response times.

\section{EFFICIENCY}

As noted by several groups before us, a clear advantage of Nexfin technology is that setup time is extremely short when compared to the multistep procedure required for arterial line placement [Stover 2009; Broch 2012; Bubenek-Turconi 2013; Berg 2014]. As such, a patient's hemodynamic profile can be obtained rather quickly upon entry into the procedure room. At our site, we have found that we can connect a patient to the ClearSight apparatus and begin data transmission within 1-2 minutes, allowing the procedure start time to be under 15 minutes after entering the operating room, compared with up to 45 minutes with the placement and calibration of arterial catheters and central venous catheters. This fast turnaround corresponds to a relative time saving of approximately $30 \mathrm{~min}$ utes per patient, creating a window for up to 2-3 additional procedures per day. This shortens waiting lists and allows greater site efficiency. Furthermore, a single team and a single operating room can be used to perform all procedures, rather than constantly switching and wasting time and resources. At our site, when fewer interventions are scheduled in one day, this frees up the hybrid OR for use by other surgical teams, further increasing the center's productivity. Importantly, the learning curve associated with the ClearSight system is also minimal, requiring only a simple setup procedure that requires little operator skill [ClearSight System Setup Guide 2013; Kuster 2015]. This is in contrast to the extensive training required for arterial cannulation, with one study reporting residents requiring an average of 20 attempts to reach a success rate of just $80 \%$ and failing to reach $100 \%$ success even after 70 procedures [Konrad 1998].

\section{SAFETY}

Besides the general discomfort related to the introduction of a foreign entity into the body, A-line placement has been associated with a number of complications. These include hematomas, abscesses, puncture site bleeding, pseudoaneurysms, air embolization, median nerve paralysis, local infection, thrombosis, arterial occlusion, and sepsis [Scheer 2002; Lorente 2006; Safdar 2013; Berg 2014; Nuttall 2016]. A recent study of 57,787 surgical patients found A-line-associated vascular complications or nerve injuries to occur at a rate of 3.6 per 10,000 patients, with this rate rising to 9.7 per 10,000 in patients undergoing cardiac procedures [Nuttall 2016]. A large-scale metaanalysis found temporary occlusion to occur at a rate of $19.7 \%$ in individuals cannulated for hemodynamic monitoring purposes, with a local infection incidence of $0.72 \%-0.78 \%$ [Scheer 2002]. Similarly, in 514 patients across intensive care units (ICU) in 6 countries monitored by a pulse contour cardiac output (PiCCO; Pulsion Medical Systems SE, Munich, Germany) device, the incidences of site inflammation and catheter-related infection were $2 \%$ and $0.78 \%$, respectively [Belda 2011]. All of the above indicate the huge potential for reducing such periprocedural complications through use of a noninvasive continuous monitoring system such as ClearSight. Given that valve interventions are generally performed in an elderly, high-risk population for whom complications would be particularly serious [Osnabrugge 2013], any procedural modifications which further reduce their likelihood is especially welcome.

\section{PATIENT SCOPE}

A-line hemodynamic monitoring is often complicated in elderly and obese patients owing to exhausted, unsuitable, or damaged vasculature and a lack of clear anatomic landmarks [Stelfox 2006; Moist 2012]. This is particularly pertinent in valve interventions, given that many patients have a high BMI and are of an advanced age. The lack of necessity for artery location and self-calibration of the ClearSight system avoid such problems. Indeed, use in obese patients is facilitated by the availability of 3 different finger-cuff sizes that allow the technology to be adapted to body habitus. As in an elderly population, diabetes is also highly prevalent in these patients [Falcao-Pires 2011]. This patient subset is particularly susceptible to infection, and the risk associated with cannulation may be higher. Noninvasive monitoring techniques may therefore be beneficial in this sense; however, a recent study showed the accuracy and precision of Nexfin technology to be negatively affected by the presence of diabetes or other vasculopathy-related conditions, such as Raynaud disease [Heusdens 2016; Alfano 2017]. Consequently, care should be taken when using the system in such patients.

\section{POTENTIAL FOR COST SAVINGS}

The initial economic outlay for the ClearSight system is not insubstantial, and a new finger cuff is required for every patient. However, when one cuff is used alone, it may serve for up to 72 hours, which is considerably longer than the periprocedural period typically surrounding valve interventions [Mayr 2015]; where longer-term monitoring is desired, use of 2 cuffs simultaneously allows switching between fingers. It 
is also worth noting that monitors for invasive hemodynamic monitoring systems are similarly expensive, that much of the apparatus is designed for single use, and that the iced solution required for thermodilution must also be purchased [Malbrain 2005]. Consequently, material costs may not be dissimilar between the 2 approaches. Furthermore, the potential for departmental cost avoidance due to time saved in preparing patients, reduced staffing requirements, and elimination of catherization-related complications is substantial with the ClearSight system. The economic implications of doing more procedures per day have not yet been formally determined.

\section{POTENTIAL LIMITATIONS OF THE CLEARSIGHT SYSTEM}

Firstly, A-line access does not merely provide BP information, but also permits blood sampling, analysis, and acid-base monitoring. The ClearSight system is unable to provide such information, though does offer a range of other parameters that are not currently available from an A-line alone.

Secondly, although our experience is that the quality of hemodynamic management during valve interventions is not adversely affected by switching to noninvasive monitoring and clinical outcomes are largely positive, this has not yet been formally evaluated. There is potential, though, for vertical repositioning of the limbs to affect pressure readings [Siddiqui 2007]. However, ClearSight is equipped with a heart reference system and automatically compensates for inaccuracies resulting from hand positioning. Furthermore, the psychological advantages of a noninvasive technique from a conscious patient's perspective should not be overlooked. Personally, we have used the ClearSight system in a large number of patients undergoing valve interventions under conscious sedation at our site and found it to be equally as efficient in this population as in general anesthesia patients. A further personal impression was patient satisfaction due to the lack of line placement pre- or intraoperatively and due to comfort postoperatively due to the lack of pain caused by an A-line or central-line/PA catheter.

Finally, the accuracy, precision, adaptability, and safety profiles of other continuous noninvasive hemodynamic monitoring approaches have not been directly compared to that of Nexfin technology during valve interventions. Though our experience is with the ClearSight system, it is possible that similar benefits may be achieved with other noninvasive systems. Comparative studies between noninvasive methods that include gold standard hemodynamic monitoring techniques as a reference and provide an analysis of relative cost-effectiveness would now be informative.

\section{CONCLUSIONS}

Although the accuracy and precision of ClearSight BP and $\mathrm{CO}$ readings are limited when compared with the gold standard A-line, we have found the biases to be tolerable during heart valve interventions. Furthermore, other factors such as safety, adaptability, applicability, and costs must also be considered when determining a system's effectiveness. Finally, better postoperative outcomes, such as shorter length of stay (LOS), decreased postoperative infection, decreased postoperative arrhythmia, decreased postoperative renal failure, decreased postoperative CHF, and decreased readmission have been observed. On balance, we consider the ClearSight system's user-friendliness, speed, potential to reduce complications, and likely cost-effectiveness to outweigh its slight inaccuracies and have thus adopted it as standard at our center. Furthermore, because many procedures are becoming increasingly simplified, we bring into question the appropriacy of invasive monitoring measures when reasonable noninvasive alternatives exist. Formal studies are now required to corroborate our observations.

\section{ACKNOWLEDGMENTS}

Peter Bramlage prepared the first draft of the article; Edwards Lifesciences funded the first-draft preparation.

\section{REFERENCES}

Alfano G, Fontana F, Cappelli G. 2017. Noninvasive blood pressure measurement in maintenance hemodialysis patients: comparison of agreement between oscillometric and finger-cuff methods. Nephron 136: 309-17.

Ameloot K, Van De Vijver K, Broch O, et al. 2013. Nexfin noninvasive continuous hemodynamic monitoring: validation against continuous pulse contour and intermittent transpulmonary thermodilution derived cardiac output in critically ill patients. ScientificWorldJournal 2013:519080.

Ameloot K, Van De Vijver K, Van Regenmortel N, et al. 2014. Validation study of Nexfin ${ }^{\circledR}$ continuous non-invasive blood pressure monitoring in critically ill adult patients. Minerva Anestesiol 80:1294-301.

Balzer F, Habicher M, Sander M, et al. 2016. Comparison of the noninvasive Nexfin ${ }^{\circledR}$ monitor with conventional methods for the measurement of arterial blood pressure in moderate risk orthopaedic surgery patients. J Int Med Res 44:832-43.

Belda FJ, Aguilar G, Teboul JL, et al. 2011. Complications related to lessinvasive haemodynamic monitoring. Br J Anaesth 106:482-6.

Berg K, Riesenberg LA, Berg D, et al. 2014. The development of a validated checklist for radial arterial line placement: preliminary results. Am J Med Qual 29:242-6.

Berkelmans GFN, Kuipers S, Westerhof BE, Spoelstra-de Man AME, Smulders YM. 2018. Comparing volume-clamp method and intraarterial blood pressure measurements in patients with atrial fibrillation admitted to the intensive or medium care unit. J Clin Monit Comput $32: 439-46$

Bland JM, Altman DG. 1986. Statistical methods for assessing agreement between two methods of clinical measurement. Lancet 1:307-10.

Bogert LW, Wesseling KH, Schraa O, et al. 2010. Pulse contour cardiac output derived from non-invasive arterial pressure in cardiovascular disease. Anaesthesia 65:1119-25.

Broch O, Bein B, Gruenewald M, et al. 2013. A comparison of continuous non-invasive arterial pressure with invasive radial and femoral pressure in patients undergoing cardiac surgery. Minerva Anestesiol 79:248-56.

Broch O, Renner J, Gruenewald M, et al. 2012. A comparison of the Nexfin ${ }^{\circledR}$ and transcardiopulmonary thermodilution to estimate cardiac output during coronary artery surgery. Anaesthesia 67:377-83. 
Bubenek-Turconi SI, Craciun M, Miclea I, Perel A. 2013. Noninvasive continuous cardiac output by the Nexfin before and after preload-modifying maneuvers: a comparison with intermittent thermodilution cardiac output. Anesth Analg 117:366-72.

Cattaneo S, Lagrotta M. 2010. Transcatheter implantation of an aortic valve: anesthesiological management. Minerva Anestesiol 76:287-9.

ClearSight System Setup Guide. c2013. Irvine (CA): Edwards Lifesciences; [accessed 2018 Aug 22]. http://ht.edwards.com/scin/edwards/ sitecollectionimages/products/mininvasive/ar10425-clearsight_ setupguide_4x6_us_1hrcrop.pdf.

de Wilde RB, de Wit F, Geerts BF, et al. 2016. Non-invasive continuous arterial pressure and pulse pressure variation measured with Nexfin ${ }^{\circledR}$ in patients following major upper abdominal surgery: a comparative study. Anaesthesia 71:788-97.

Dolmatova E, Moazzami K, Cocke TP, et al. 2017. Extracorporeal membrane oxygenation in transcatheter aortic valve replacement. Asian Cardiovasc Thorac Ann 25:31-4.

Falcao-Pires I, Hamdani N, Borbely A, et al. 2011. Diabetes mellitus worsens diastolic left ventricular dysfunction in aortic stenosis through altered myocardial structure and cardiomyocyte stiffness. Circulation 124:1151-9.

Fassl J, Walther T, Groesdonk HV, et al. 2009. Anesthesia management for transapical transcatheter aortic valve implantation: a case series. J Cardiothorac Vasc Anesth 23:286-91.

Fischer MO, Avram R, Cârjaliu I, et al. 2012. Non-invasive continuous arterial pressure and cardiac index monitoring with Nexfin after cardiac surgery. Br J Anaesth 109:514-21.

Giustino G, Montorfano M, Chieffo A, et al. 2014. TCT-776 Risk factors and clinical significance of intra-procedural haemodynamic instability in patients undergoing transcatheter aortic valve implantation. JACC 64(11 suppl):B227.

Heusdens JF, Lof S, Pennekamp CW, et al. 2016. Validation of noninvasive arterial pressure monitoring during carotid endarterectomy. $\mathrm{Br}$ J Anaesth 117:316-23.

Hofhuizen C, Lansdorp B, van der Hoeven JG, Scheffer GJ, Lemson J. 2014. Validation of noninvasive pulse contour cardiac output using finger arterial pressure in cardiac surgery patients requiring fluid therapy. J Crit Care 29:161-5.

Hohn A, Defosse JM, Becker S, Steffen C, Wappler F, Sakka SG. 2013. Non-invasive continuous arterial pressure monitoring with Nexfin does not sufficiently replace invasive measurements in critically ill patients. $\mathrm{Br}$ J Anaesth 111:178-84.

Joosten A, Desebbe O, Suehiro K, et al. 2017. Accuracy and precision of non-invasive cardiac output monitoring devices in perioperative medicine: a systematic review and meta-analysis. Br J Anaesth 118:298-310.

Kalmar AF, Vos JJ, Weening M, et al. 2012. Validation of continuous noninvasive arterial blood pressure measurements during general anesthesia. Presented at: ANESTHESIOLOGYTM 2012 Annual Meeting; Washington, DC. Abstract 174.

Kalmar AF, Wesseling W, Scheeren TWL. 2013. Technology report: ccNexfin monitor. Nederlands Tijdschrift voor Anesthesiologie 26:3-7.

Konrad C, Schupfer G, Wietlisbach M, Gerber H. 1998. Learning manual skills in anesthesiology: is there a recommended number of cases for anesthetic procedures? Anesth Analg 86:635-9.

Kuster M, Exadaktylos A, Schnuriger B. 2015. Non-invasive hemodynamic monitoring in trauma patients. World J Emerg Surg 10:11.
Landes U, Barsheshet A, Finkelstein A, et al. 2017. Temporal trends in transcatheter aortic valve implantation, 2008-2014: patient characteristics, procedural issues, and clinical outcome. Clin Cardiol 40:82-8.

Lee C, Casserly I, Fahy E. 2017. 43 Comparing outcomes in patients undergoing tavi: general anaesthetic versus conscious sedation. Heart 103:A25-6.

Lorente L, Santacreu R, Martín MM, Jiménez A, Mora ML. 2006. Arterial catheter-related infection of 2,949 catheters. Crit Care 10:R83.

Maass SW, Roekaerts PM, Lance MD. 2014. Cardiac output measurement by bioimpedance and noninvasive pulse contour analysis compared with the continuous pulmonary artery thermodilution technique. J Cardiothorac Vasc Anesth 28:534-9.

Maggi R, Viscardi V, Furukawa T, Brignole M. 2010. Non-invasive continuous blood pressure monitoring of tachycardic episodes during interventional electrophysiology. Europace 12:1616-22.

Malbrain MLNG, De Potter TJR, Deeren D. 2005. Cost-effectiveness of minimally invasive hemodynamic monitoring. In: Vincent JL, editor. Yearbook of intensive care and emergency medicine. New York (NY): Springer. p 603-31.

Martina JR, Westerhof BE, Van Goudoever J, et al. 2010. Noninvasive blood pressure measurement by the Nexfin monitor during reduced arterial pulsatility: a feasibility study. ASAIO J 56:221-7.

Martina JR, Westerhof BE, van Goudoever J, et al. 2012. Noninvasive continuous arterial blood pressure monitoring with Nexfin ${ }^{\circledR}$. Anesthesiology 116:1092-103.

Mayr NP, Michel J, Bleiziffer S, Tassani P, Martin K. 2015. Sedation or general anesthesia for transcatheter aortic valve implantation (TAVI). J Thorac Dis 7:1518-26.

Moist LM, Lok CE, Vachharajani TJ, et al. 2012. Optimal hemodialysis vascular access in the elderly patient. Semin Dial 25:640-8.

Monnet X, Picard F, Lidzborski E, et al. 2012. The estimation of cardiac output by the Nexfin device is of poor reliability for tracking the effects of a fluid challenge. Crit Care 16:R212.

Nuttall G, Burckhardt J, Hadley A, et al. 2016. Surgical and patient risk factors for severe arterial line complications in adults. Anesthesiology 124:590-7.

O'Brien E, Atkins N, Stergiou G, et al. 2010. European Society of Hypertension International Protocol revision 2010 for the validation of blood pressure measuring devices in adults. Blood Press Monit 15:23-38.

Osnabrugge RLJ, Mylotte D, Head SJ, et al. 2013. Aortic stenosis in the elderly: disease prevalence and number of candidates for transcatheter aortic valve replacement: a meta-analysis and modeling study. J Am Coll Cardiol 62:1002-12.

Peter L, Noury N, Cerny M. 2014. A review of methods for non-invasive and continuous blood pressure monitoring: pulse transit time method is promising? IRBM 35:271-82.

Petzoldt M, Riedel C, Braeunig J, et al. 2015. Dynamic device properties of pulse contour cardiac output during transcatheter aortic valve implantation. J Clin Monit Comput 29:323-31.

Raggi EP, Sakai T. 2017. Update on finger-application-type noninvasive continuous hemodynamic monitors (CNAP and ccNexfin): physical principles, validation, and clinical use. Semin Cardiothorac Vasc Anesth 21:321-9.

Safdar N, O’Horo JC, Maki DG. 2013. Arterial catheter-related bloodstream infection: incidence, pathogenesis, risk factors and prevention. J Hosp Infect 85:189-95. 
Scheer BV, Perel A, Pfeiffer UJ. 2002. Clinical review: complications and risk factors of peripheral arterial catheters used for haemodynamic monitoring in anaesthesia and intensive care medicine. Crit Care 6:199-204.

Schraverus P, Kuijpers MM, Coumou J, Boly CA, Boer C, van Kralingen S. 2016. Level of agreement between cardiac output measurements using Nexfin ${ }^{\circledR}$ and thermodilution in morbidly obese patients undergoing laparoscopic surgery. Anaesthesia 71:1449-55.

Siddiqui F, Strus J, Ming X, Lee IA, Chokroverty S, Walters AS. 2007. Rise of blood pressure with periodic limb movements in sleep and wakefulness. Clin Neurophysiol 118:1923-30.

Sperna Weiland NH, de Wever JW, van Duivenvoorde Y, et al. 2018. Agreement between ccNexfin CO-trek cardiac output and intermittent cold-bolus pulmonary thermodilution in a prospective multicenter study. Minerva Anestesiol 84:473-80.

Stelfox HT, Ahmed SB, Ribeiro RA, Gettings EM, Pomerantsev E, Schmidt U. 2006. Hemodynamic monitoring in obese patients: the impact of body mass index on cardiac output and stroke volume. Crit Care Med 34:1243-6.

Stenglova A, Benes J. 2017. Continuous non-invasive arterial pressure assessment during surgery to improve outcome. Front Med (Lausanne) 4:202.

Stover JF, Stocker R, Lenherr R, et al. 2009. Noninvasive cardiac output and blood pressure monitoring cannot replace an invasive monitoring system in critically ill patients. BMC Anesthesiol 9:6.

Tamburino C, Capodanno D, Ramondo A, et al. 2011. Incidence and predictors of early and late mortality after transcatheter aortic valve implantation in 663 patients with severe aortic stenosis. Circulation 123:299-308.

Van de Vijver K, Verstraeten A, Gillebert C, et al. 2011. Validation of non-invasive hemodynamic monitoring with Nexfin in critically ill patients. Crit Care 15(suppl 1):P75.

Vos JJ, Poterman M, Mooyaart EA, et al. 2014. Comparison of continuous non-invasive finger arterial pressure monitoring with conventional intermittent automated arm arterial pressure measurement in patients under general anaesthesia. Br J Anaesth 113:67-74

Weiss E, Gayat E, Dumans-Nizard V, Le Guen M, Fischler M. 2014. Use of the Nexfin device to detect acute arterial pressure variations during anaesthesia induction. Br J Anaesth 113:52-60. 